\title{
An Experimental Investigation on Energy Performance of The Hybrid Photovoltaic Thermal System
}

\author{
Shahrokh Barati ${ }^{1, *}$, Livio de Santoli ${ }^{1}$,Gianluigi Lo Basso ${ }^{1}$, Antonio Galizia ${ }^{1}$, Giulia \\ Spiridigliozzi ${ }^{1}$
}

${ }^{I}$ DIAEE-Department of Astronautics Electrical Energy Engineering, Sapienza, University of Rome, Via Eudossiana 18, Rome 00184, Italy

\begin{abstract}
Climate change is a worldwide recognized problem, and its mitigation identified as one of the most significant challenges. The way to achieve this purpose is to reduce greenhouse gases (GHG) emissions through the energy system using renewables. The change from an energy system based on fossil fuels to renewable sources-based one is necessary on which the world community agrees. A photovoltaic thermal (PV/T) panel is a system that can produce both electricity and thermal energy simultaneously in one integrated system. This paper deals with hybrid energy systems, specifically a hybrid system to produce power and thermal energy from solar sources consisting of photovoltaic thermal modules. The hybrid system consists of 7 hybrid photovoltaic panels installed on the roof of the laboratory. This paper presents a study for experimental data obtained from a measurement campaign of the thermal and electrical behavior of a PV/T system in single and series models.
\end{abstract}

\section{Introduction}

In the last decades, there is a critical desire to change the energy supply, since this involves a decrease of the emissions associated with fossil fuel that leads to more extensive environmental and health concerns. PV is one of the most extensively utilized renewable power sources all around the world. Currently, the development of PV investments has been remarkable [1-3]. Moreover, the most significant challenge development of the solar photovoltaic industry is High production cost and Low efficiency of photovoltaic power generation. Commercial PV electrical conversion efficiency is of 6 to 15\%.[4] Improving the efficiency performance of solar energy collection by developing a hybrid photovoltaic/thermal solar collector has been investigated by several researchers [5-10]. Herrando et al. [11] have analyzed the energy and economic performance of PV/T used to provide the energy demands of single-family indicating homes in three European cities,

\footnotetext{
${ }^{*}$ Corresponding author : Shahrokh.barati@uniroma1.it
} 
This research highlights that these systems can provide about $65 \%$ of electricity requirements and between $30 \%$ and $60 \%$ of thermal demands. Van Helden et al. [12] Carried out that PV/T modules' total efficiency is higher than the sum of the separate PV and solar thermal systems. PV/T can add to the decrease in fossil fuel consumption in the built environment in a more cost-effective approach. Hegazy et al. [13] investigated a thermal, electrical, hydraulic, and overall design of four types of PV/T air collectors. The numerical analysis described that while the channel above PV mode has the lowest performance, the other three have similar energy yields. Also, single-pass channels with PV in-between consume the slightest fan power. Alzaabi et al. [14] Presented a design to develop a PV panel's electrical efficiency utilizing a water hybrid PV/T system. The system made of a polycrystalline PV panel with a solar thermal collector adhered to its backside. The outcomes confirmed that the PV/T system's electrical power output increased by 15$20 \%$ compared to the PV panel. The system's thermal efficiency was calculated from measured data and reached values close to $60 \%-70 \%$ was achieved. Bahaidarah et al. [15] described the water PV/T system's effectiveness in the hot weather area of Dhahran, Saudi Arabia. Consequences demonstrated that PV temperatures decreased by $20.0 \%$ using a water-cooling system, and PV efficiency is increased by $9.0 \%$. The present work focuses on a hybrid PV/T performance in the laboratory at Sapienza University. The hybrid system consists of 7 hybrid photovoltaic panels installed on the roof of the laboratory. The panels are electrically installed in series, the previous studies did not compare to series, and a single photovoltaic/thermal system include experimental or numerical investigations from the electrical and thermal energy. This research aims to estimate the electrical and thermal production in two different modes for the dwelling.

\section{System description}

This research consists of seven PV panels installed on the laboratory's rooftop at the Sapienza University. The schematic of the experimental campaign of PV panels is presented in Fig.1. As indicated in fig.2. The back of the board features the aluminum coil that captures the thermal energy. The dimensions of the hybrid panel are standard 1000x1652, precisely like any conventional photovoltaic panel. Electrical and thermal characterization are shown in Tables 1 and 2, respectively.

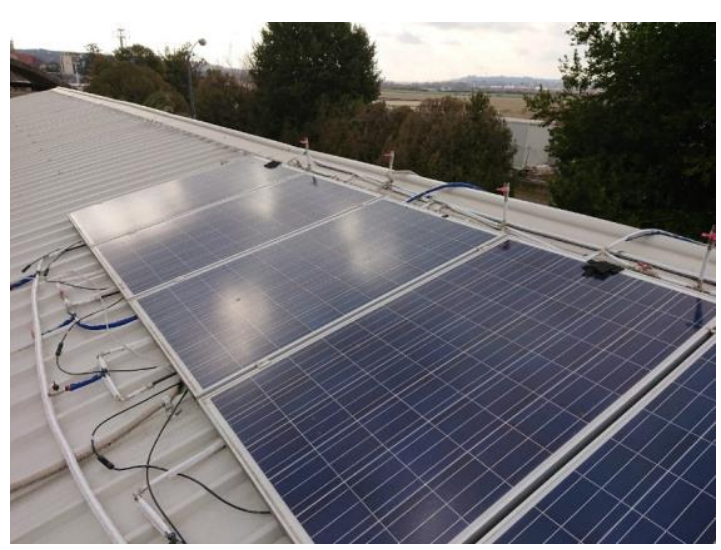

Fig. 1. General view of the PV panels.

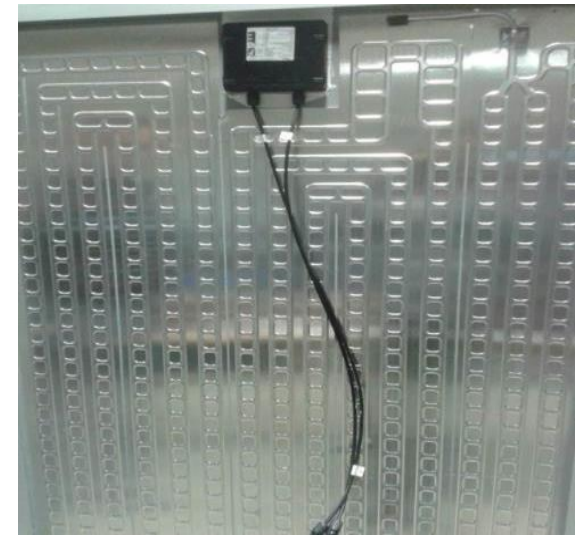

Fig. 2. Plate heat exchanger PV 


\subsection{Electrical characteristics}

Table 1 presents the electrical characteristics of the panel declared by the manufacturer.

Table 1. Electrical Parameters

\begin{tabular}{|l|c|}
\hline Peak power Pm(W) & $230 \mathrm{~W}$ \\
\hline Voltage MPP Vm(V) & 30 \\
\hline Current MPP Im(A) & 7.67 \\
\hline Open circuit voltage Voc (V) & 36.8 \\
\hline Closed circuit current Isc (A) & 8.34 \\
\hline Maximum system voltage (VDC) & 1000 \\
\hline Panel efficiency $(\%)$ & $13.9 \%$ \\
\hline Maximum surface load & $2400 \mathrm{~N} / \mathrm{m}$ \\
\hline power temperature $\mathrm{W} \%{ }^{\circ} \mathrm{C}$ & $-0.45 \% /{ }^{\circ} \mathrm{C}$ \\
\hline voltage temperature $\operatorname{Voc} \%{ }^{\circ} \mathrm{C}$ & $-0.36 \% /{ }^{\circ} \mathrm{C}$ \\
\hline current temperature $\operatorname{Isc} \%{ }^{\circ} \mathrm{C}$ & $+0.05 \% /{ }^{\circ} \mathrm{C}$ \\
\hline
\end{tabular}

\subsection{Thermal characteristics}

The thermal characteristics were taken directly from the manufacturer's data.

Table 2. Thermal Parameters

\begin{tabular}{|l|c|}
\hline Load loss & $150 \mathrm{mbar}$ \\
\hline Maximum allowed operating temperature & $80{ }^{\circ} \mathrm{C}$ \\
\hline Maximum operating pressure & $3 \mathrm{bar}$ \\
\hline Recommended flow rate & $1,2 \mathrm{l} / \mathrm{min}$ \\
\hline Volume of fluid in the panel & 0,81 \\
\hline surface area & $1,52 \mathrm{~m} 2$ \\
\hline Absorber surface & $1,46 \mathrm{~m} 2$ \\
\hline Optical efficiency & $\eta=0,513$ \\
\hline Linear heat loss coefficient & $\mathrm{a} 1=7,680 \mathrm{~W} /\left(\mathrm{m} 2^{\circ} \mathrm{K}\right)$ \\
\hline Quadratic heat loss coefficient & $\mathrm{a} 2=0,014 \mathrm{~W} /\left(\mathrm{m} 2{ }^{\circ} \mathrm{K} 2\right)$ \\
\hline
\end{tabular}

\section{Experimental procedures}

The following methods were conducted from 11 AM to 4 PM for the seven panels. In the single and series model, the results were recorded every 30 minutes. The system is installed on the roof, connected with the detects solar radiation result carried out by entering the panel data. The sampling of the data Are as follows:

1. The water flow rate in the circuit $[\mathrm{m} 3 / \mathrm{h}]$

2. Input and output temperature (Tin and Tout exchanger $\left[{ }^{\circ} \mathrm{C}\right]$ )

3. $\Delta \mathrm{T}$ Primary hydraulic circuit

4. Thermal power [W]

5. Solar irradiation $[\mathrm{W} / \mathrm{m} 2]$, inclination $\beta=23^{\circ}$, azimuth angle $=50^{\circ} \mathrm{N}, \mathrm{f}$ ) Solar cell temperature $\mathrm{TPV}\left[{ }^{\circ} \mathrm{C}\right]$

6. Voltage [V]

7. Current $[\mathrm{A}]$ 
The characterization of the hybrid PV/T solar module was conducted by evaluating the parameters from which it can recognize the thermal, electrical, and first principle efficiency within a parametric investigation. However, we studied calibrating the characterization of parameters that most influence a PV/T hybrid solar collector's operational operation. We constructed a corrected $\Delta T^{*}$, which was an expression of the temperature difference between the photovoltaic plate $\mathrm{Tpv}$ and the average of the fluid in the printed plate heat exchanger, normalized on the incident radiation. It was possible to achieve the equations of the efficiency characteristic curves that characterize the PV/T. The purpose of this investigation is to see what happens by setting several modules in series.

\subsection{Evaluation of the electric efficiency $\eta_{\mathrm{el}}$}

1) Electrical efficiency of the solar module, such as:

$$
\eta_{e l}=\frac{P_{e l}}{G_{t}}
$$

2) $\Delta T^{*}$ correct, i.e. normalized on radiation:

$$
\Delta T^{*}=\frac{\left(T_{p v}-T_{m}\right)}{G_{t}}
$$

Then

$$
\left.\eta_{e l}=-10846 \Delta T^{*^{3}}+150.28 \Delta T^{*^{2}}+2.2329 \Delta T^{*}+0.1343 \quad\right) 3(
$$

\subsection{Evaluation of the thermal recovery efficiency $\eta_{t}$}

1) Thermal efficiency of the solar module, such as:

$$
\eta_{t}=\frac{P_{t}}{G_{t}}
$$

2) $\Delta T^{*}$ corrected, i.e. normalized on radiation

$$
\Delta T^{*}=\frac{\left(T_{p v}-T_{m}\right)}{G_{t}}
$$

Then

$$
\left.\eta_{t}=-320732 \Delta T^{*^{3}}+12167 \Delta T^{*^{2}}-142.68 \Delta T^{*}+1.2589 \quad\right) 6(
$$

\subsection{Performance evaluation of first principle $\eta_{c o g}$}

The First Principle Efficiency curve corresponds to the point-by-point sum of the $\eta \_t$ and $\eta \_$el curves as a function $\Delta T^{*}$ :

$$
\eta_{\operatorname{cog}}=\eta_{e l}+\eta_{t}
$$

The interpolated curve has an even maximum point $\eta_{\operatorname{cog}}=0941$ around $\Delta T^{*}=0.016 \mathrm{~S}$ 


\section{Result and discussion}

The result parameters single panel data, as illustrated in Table 3, also. Fig.3 is shown the parametric curves thermal and electrical from experimental data for a single panel.

Table 3. Single panel data used for comparison with the 3 panels series

\begin{tabular}{|c|c|c|c|c|c|c|c|c|c|c|}
\hline $\begin{array}{c}\text { HOURS } \\
{[\mathrm{h}]}\end{array}$ & $\begin{array}{c}\text { FLOW } \\
{[\mathrm{m} 3 / \mathrm{h}]}\end{array}$ & $\begin{array}{c}\text { Tin } \\
{\left[{ }^{\circ} \mathrm{C}\right]}\end{array}$ & $\begin{array}{c}\text { Tout } \\
{\left[{ }^{\circ} \mathrm{C}\right]}\end{array}$ & $\begin{array}{c}\Delta \mathrm{T} \\
{\left[{ }^{\circ} \mathrm{C}\right]}\end{array}$ & $\begin{array}{c}\text { Thermal } \\
\text { power } \\
{[\mathrm{W}]}\end{array}$ & $\begin{array}{c}\text { Irr. } \\
{[\mathrm{W} / \mathrm{m} 2]}\end{array}$ & $\begin{array}{c}\mathrm{Tpv} \\
{\left[{ }^{\circ} \mathrm{C}\right]}\end{array}$ & $\begin{array}{c}\mathrm{V} \\
{[\text { volt }]}\end{array}$ & $\begin{array}{c}\text { I } \\
{[\text { Ampere }]}\end{array}$ & $\begin{array}{c}\text { Electric } \\
\text { power } \\
{[\mathrm{W}]}\end{array}$ \\
\hline 11.00 & 0.209 & 36.5 & 33.3 & 3.2 & 802 & 670 & 47.5 & 25.58 & 6.57 & 168.1 \\
\hline 11.30 & 0.21 & 37.4 & 34.4 & 3 & 730 & 673 & 47.6 & 25.52 & 7.02 & 179.2 \\
\hline 12.00 & 0.21 & 37.8 & 34.5 & 3.3 & 760 & 684 & 47.8 & 25.58 & 6.83 & 174.7 \\
\hline 12.30 & 0.209 & 38.2 & 34.8 & 3.4 & 807 & 690 & 48 & 27.3 & 6.01 & 164.1 \\
\hline 13.00 & 0.209 & 37.2 & 35.1 & 2.1 & 436 & 369 & 46.8 & 28.4 & 3.52 & 100.0 \\
\hline 13.30 & 0.211 & 38.8 & 35.7 & 3.1 & 783 & 701 & 47.9 & 25.08 & 7.12 & 178.6 \\
\hline 14.00 & 0.212 & 39 & 36 & 3 & 730 & 672 & 47.8 & 25.5 & 6.96 & 177.5 \\
\hline 14.30 & 0.21 & 39.5 & 36.7 & 2.8 & 742 & 679 & 47.9 & 26.66 & 5.64 & 150.4 \\
\hline 15.00 & 0.212 & 39.3 & 36.4 & 2.9 & 714 & 583 & 45.5 & 27.3 & 5.26 & 143.6 \\
\hline 15.30 & 0.212 & 39.2 & 36.7 & 2.5 & 617 & 452 & 43.9 & 27.4 & 4.45 & 121.9 \\
\hline 16.00 & 0.211 & 39 & 36.7 & 2.3 & 575 & 401 & 42.2 & 27.87 & 3.82 & 106.5 \\
\hline
\end{tabular}

Table 4. Parametric analysis of for a single panel

\begin{tabular}{|c|c|c|}
\hline Ren.Term & Ren.El & $\begin{array}{c}\text { Ren. } \\
\text { First Principle }\end{array}$ \\
\hline 0.7388981 & 0.154837479 & 0.893735581 \\
\hline 0.66956506 & 0.16431897 & 0.833884028 \\
\hline 0.68587106 & 0.157670385 & 0.843541441 \\
\hline 0.72195384 & 0.146782072 & 0.86873591 \\
\hline 0.72936532 & 0.167232092 & 0.89659741 \\
\hline 0.6894912 & 0.157244149 & 0.846735352 \\
\hline 0.67056143 & 0.163029101 & 0.833590535 \\
\hline 0.67455772 & 0.136695576 & 0.811253296 \\
\hline 0.75598755 & 0.152042437 & 0.908029985 \\
\hline 0.84261991 & 0.166516443 & 1.009136349 \\
\hline 0.88513285 & 0.163885656 & 1.049018503 \\
\hline
\end{tabular}

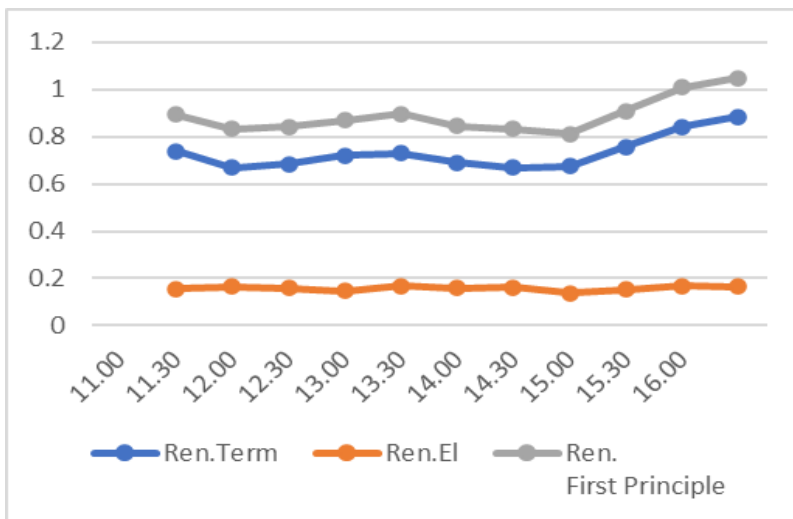

Fig 3. Parametric curves from experimental data for single panel 
Table 5 presents the investigated result experimental campaign series form of three PV/T panels, Fig. 4 reveals a comparison for parametric performance curves from experimental data for the series of 3 panels.

Table 5. Series data from 3 panels used to compare the single panel

\begin{tabular}{|c|c|c|c|c|c|c|c|c|c|r|r|r|}
\hline $\begin{array}{c}\text { HOURS } \\
{[\mathrm{h}]}\end{array}$ & $\begin{array}{c}\text { FLOW } \\
{[\mathrm{m} 3 / \mathrm{h}]}\end{array}$ & $\begin{array}{c}\text { Tin } \\
{\left[{ }^{\circ} \mathrm{C}\right]}\end{array}$ & $\begin{array}{c}\text { Tout } \\
{\left[{ }^{\circ} \mathrm{C}\right]}\end{array}$ & $\begin{array}{c}\Delta \mathrm{T} \\
{\left[{ }^{\circ} \mathrm{C}\right]}\end{array}$ & $\begin{array}{c}\text { Thermal } \\
\text { power } \\
{[\mathrm{W}]}\end{array}$ & $\begin{array}{c}\text { Irr. } \\
{[\mathrm{W} / \mathrm{m} 2]}\end{array}$ & $\begin{array}{l}\text { Tpv1 } \\
{\left[{ }^{\circ} \mathrm{C}\right]}\end{array}$ & $\begin{array}{l}\text { Tpv2 } \\
{\left[{ }^{\circ} \mathrm{C}\right]}\end{array}$ & Tpv3 $\left[{ }^{\circ} \mathrm{C}\right]$ & $\begin{array}{l}\mathrm{V} \\
{[\text { volt }]}\end{array}$ & $\begin{array}{l}\text { I } \\
{[\text { Ampere }}\end{array}$ & $\begin{array}{l}\text { Electric } \\
\text { power } \\
{[\mathrm{W}]}\end{array}$ \\
\hline 11.00 & 0.14 & 43.4 & 33.7 & 9.7 & 1586 & 622 & 64.8 & 48.3 & 61.8 & 75.46 & 6.32 & 476.9 \\
\hline 11.30 & 0.14 & 44.6 & 34.7 & 9.9 & 1637 & 646 & 62.8 & 46.5 & 57.1 & 74.53 & 6.59 & 491.2 \\
\hline 12.00 & 0.14 & 45.7 & 35.8 & 9.9 & 1577 & 645 & 66.6 & 51.3 & 60.5 & 75.82 & 6.5 & 492.8 \\
\hline 12.30 & 0.141 & 46.1 & 36.5 & 9.6 & 1595 & 623 & 65.5 & 48.1 & 59.8 & 75.1 & 6.55 & 491.9 \\
\hline 13.00 & 0.142 & 46 & 36.8 & 9.2 & 1476 & 632 & 64.1 & 47.8 & 58.6 & 76.68 & 6.3 & 483.1 \\
\hline 13.30 & 0.14 & 45.8 & 37.4 & 8.4 & 1385 & 616 & 61.8 & 47.5 & 57.4 & 77.25 & 6.06 & 468.1 \\
\hline 14.00 & 0.14 & 45.7 & 37.9 & 7.8 & 1255 & 489 & 59.2 & 47.3 & 53.9 & 77.32 & 5.05 & 390.5 \\
\hline 14.30 & 0.142 & 45.5 & 38.4 & 7.1 & 1115 & 563 & 57.1 & 46.9 & 52.9 & 77.6 & 5.59 & 433.8 \\
\hline 15.00 & 0.14 & 44.5 & 38.1 & 6.4 & 1041 & 516 & 54.2 & 46.1 & 50.3 & 79.75 & 5.14 & 409.9 \\
\hline 15.30 & 0.142 & 44.2 & 38.1 & 6.1 & 1003 & 471 & 54.3 & 46.5 & 50.2 & 79.96 & 4.72 & 377.4 \\
\hline 16.00 & 0.14 & 42.5 & 37.3 & 5.2 & 835 & 409 & 45.3 & 46.1 & 50.8 & 80.39 & 4.12 & 331.2 \\
\hline
\end{tabular}

Table 6. Parametric analysis of series of 3 panels

\begin{tabular}{|c|c|c|}
\hline Ren.Term & Ren.El & $\begin{array}{c}\text { Ren. } \\
\text { First Principle }\end{array}$ \\
\hline 0.524658277 & 0.157763752 & 0.682422029 \\
\hline 0.521410644 & 0.156439979 & 0.677850622 \\
\hline 0.503078445 & 0.157217597 & 0.660296041 \\
\hline 0.526788604 & 0.162463917 & 0.689252522 \\
\hline 0.480543835 & 0.157278481 & 0.637822316 \\
\hline 0.462628935 & 0.15637025 & 0.618999185 \\
\hline 0.528078635 & 0.164300201 & 0.692378836 \\
\hline 0.40750243 & 0.158536354 & 0.566038784 \\
\hline 0.415111494 & 0.163458624 & 0.578570118 \\
\hline 0.438171127 & 0.164876063 & 0.60304719 \\
\hline 0.42007506 & 0.16662481 & 0.503078445 \\
\hline
\end{tabular}

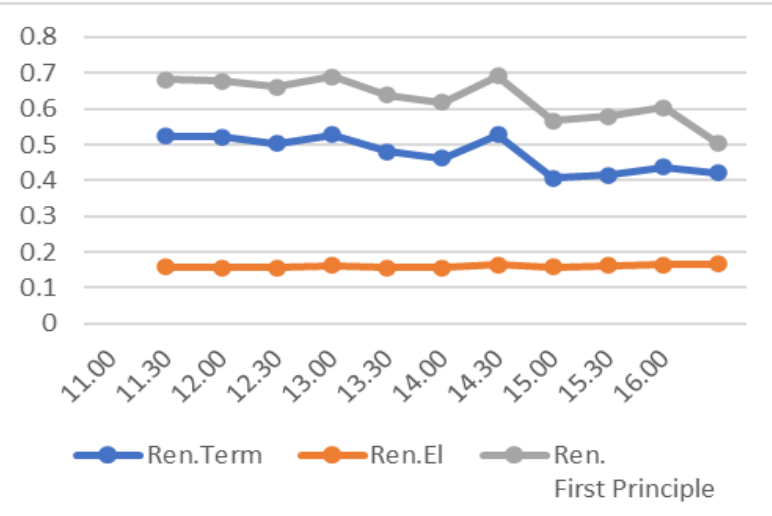

Fig 4. Parametric performance curves from experimental data for the series of 3 panels 
By highlighting the first-principle results, we can analyze the differences: Fig .5 reveals a comparison for the first principle of the single and three panels in the PV/T. It is clearly shown that the first principle performance is significantly higher in the single module compared with other works.

Table 7. Difference between First principle returns of the single panel and the series of 3

\begin{tabular}{|l|l|l|}
\hline Ren. Single panel & Ren Three panels & difference \\
\hline 0.85412663 & 0.682422029 & 17.1704601 \\
\hline 0.833884028 & 0.677850622 & 15.60334053 \\
\hline 0.843541441 & 0.660296041 & 18.32454 \\
\hline 0.86873591 & 0.689252522 & 17.94833882 \\
\hline 0.83269854 & 0.637822316 & 19.4876224 \\
\hline 0.846735352 & 0.618999185 & 22.77361666 \\
\hline 0.87425694 & 0.692378836 & 18.18781035 \\
\hline 0.811253296 & 0.566038784 & 24.52145115 \\
\hline 0.908029985 & 0.578570118 & 32.9459867 \\
\hline 1.009136349 & 0.60304719 & 40.60891591 \\
\hline 1.049018503 & 0.58669987 & 46.23186329 \\
\hline 0.85412663 & 0.682422029 & 17.1704601 \\
\hline 0.833884028 & 0.677850622 & 15.60334053 \\
\hline & Medium difference \% & 24.89126781 \\
\hline
\end{tabular}

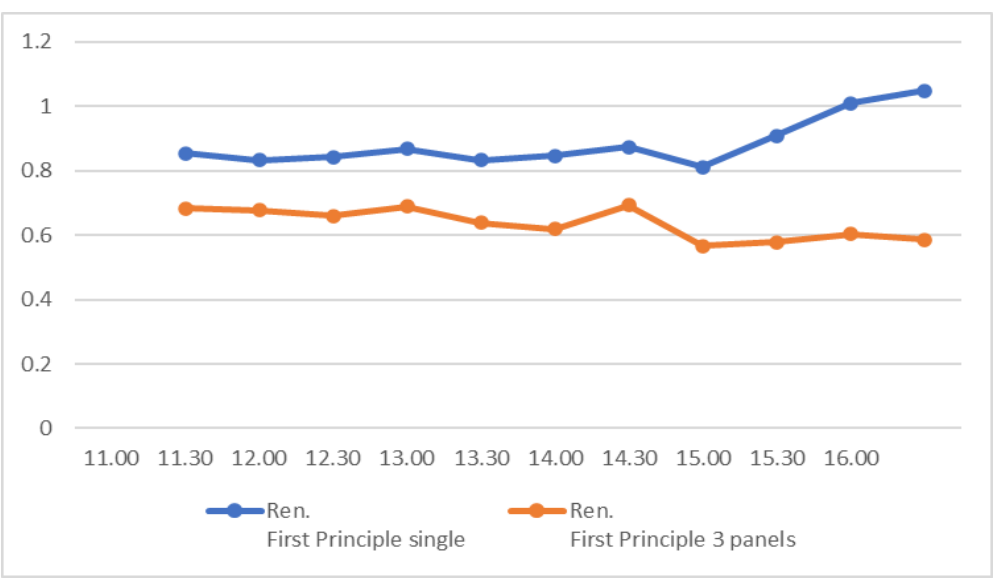

Fig 5. Difference between the first principle curves of a single panel and the series of 3 panels 


\section{Conclusions}

This work's purpose was to conduct an experimental measurement project to characterize a hybrid PV/T solar panel's real operating conditions in single module configurations and three modules connected in electric hydraulic series. From the data obtained experimentally, we analyzed the behavior of the two types of designs, from which we could observe that:

1-Single panel works with optimal efficiency, providing an increase in the thermal level of the average temperature Tm (in the summer operating period), which infrequently exceeded $40^{\circ} \mathrm{C}$, the solar cell temperature $\mathrm{Tpv}$ consequently lowers its thermal level changing between 40 and $50^{\circ} \mathrm{C}$, the lowering of these temperatures allows the cell to perform at the point of most significant electrical and thermal efficiency.

2-Series of three panels can increase the average circuit temperature Tm (compared to the previous case). However, the average thermal system always below $43^{\circ} \mathrm{C}$, compared to a solar cell temperature Tpv, which exceeds $60^{\circ} \mathrm{C}$.

Consequently, the results can also be used to evaluate the advantages of deriving by installing PV/T plants compared with conventional PV solar systems.

\section{References}

1. S. Daisuke, Y. Noboru, Renew Sustain Energy Rev 104, 151-166 (2019).

2. L. De Santoli, G. Lo Basso, Sh. Barati, S. D’ambra ,C. Fasolilli, Energy 193, 116678 (2020).

3. A. Gagliano, G.M. Tina, S. Aneli, S. Nizetic, Cleaner Prod, 304-315 (2019)

4. B.J. Huang, T.H. Lin, W.C. Hung, F.S. Sun. Energy 70, 443-448 (2001).

5. A.S. Joshi, A. Tiwari, Renew Energy 32, 2223-2241 (2007).

6. T. Ma, H. Yang, Y. Zhang, L. Lu, X. Wang Renew. Sustain. Energy Rev 4, 1273-1284 (2015).

7. N. Marc-Alain Mutombo, F. Inambao, G. Bright, Energy Southern Africa 27 (1), 28-38 (2016).

8. G. Mittelman, A. Kribus, A. Dayan, Energy Convers. Manage 48 (9), 2481 2490 (2007).

9. T. Nualboonrueng, P. Tuenpusa, Y. Ueda, A. Akisawa, Res. Appl 21, 1204-1213 (2013).

10. B. Sandnes, J. Rekstad, Energy 72 (1), 63-73 (2002).

11. M. Herrando, A. Ramosa, J. Freeman, I. Zabalza, C.N. Markides, Energy Convers Manage 175 (1), 67-85 (2018).

12. Van Helden, R.J.C. van Zolingen, H.A. Zondag,: Res. Appl 12, 415-426 (2004). 
13. A.A. Hegazy, S 861-881 (2000).

14. A. Alzaabi, Nadine K. Badawiyeh, Hind O. Hantoush, A.K. Hamid, Energy, 3853892014 (2014).

15. H. Bahaidaraha, A. Subhana, P. Gandhidasana, Energy 59, 445-453 (2013). 